\title{
Three-Dimensional On-Chip Inductance and Resistance Extraction
}

\author{
Alexandre Nentchev and Siegfried Selberherr \\ Institute for Microelectronics, Technical University Vienna, Austria \\ \{nentchev|selberherr\}@iue.tuwien.ac.at
}

\begin{abstract}
An efficient three-dimensional finite element frequency domain method to extract the inductance and resistance of small structures like on-chip interconnects or on-chip inductors is presented. Skin effect and proximity effect are taken into account. The parameters are obtained from the field energy calculated from the magnetic field distribution in the simulation domain. The small dimensions of the domain of interest provide the opportunity of using the optimized model of dominant magnetic field even at very high operating frequencies. Vector and scalar shape functions are used for finite element equation system assembling. Series of simulations for an instancing on-chip inductor at frequencies between $1 \mathrm{MHz}$ and $100 \mathrm{GHz}$ are performed to extract the parameters and to visualize the field distributions in the simulation area.
\end{abstract}

\section{Categories and Subject Descriptors}

G.1.8 [Mathematics of Computing]: Numerical analysis-Partial Differential Equations;

I.6.4 [Computing Methodologies]: Simulation and modeling-Model Validation and Analysis

\section{General Terms}

Algorithms, Reliability, Theory, Verification

\section{INTRODUCTION}

High frequencies in an integrated circuit (IC) affect both, the resistance and the inductance of the on-chip interconnects. These often as parasitics treated parameters cause longer signal rise, fall, and delay times and limit the maximum allowed frequency of modern ICs. However, as the operating frequencies increase, small inductors of high speed circuits can be also actively used. They can be even constructed on the chip. Thus the inductance of an on-chip interconnect line can be a disadvantage or very useful depending on the application. Of course the collateral resis-

Permission to make digital or hard copies of all or part of this work for personal or classroom use is granted without fee provided that copies are not made or distributed for profit or commercial advantage and that copies bear this notice and the full citation on the first page. To copy otherwise, to republish, to post on servers or to redistribute to lists, requires prior specific permission and/or a fee.

SBCCI'07, September 3-6, 2007, Rio de Janeiro, Brazil

Copyright 2007 ACM 978-1-59593-816-9/07/0009 ...\$5.00. tance must also be considered. In each case it is necessary to investigate the structure of interest to obtain its inductance and resistance in order to estimate the impact on the entire electric circuit [1]. In the case of applications in radio frequency $(\mathrm{RF}) \mathrm{ICs}$ such as voltage controlled oscillators or low noise amplifiers the inductance and the resistance of the on-chip inductors must be extensively investigated for the $\mathrm{RF}$ circuit design, performance optimization, and inductor quality factor. The frequency dependent inductance and resistance of wide on-chip interconnects must be captured to obtain the impact on power supply stability and signal delay.

Currently there are two major techniques for modeling of on-chip inductors - analytical compact modeling and numerical field calculation based modeling. In the case of a spiral inductor, where the models can be restricted to specific geometry classes, closed-form analytical models are very well suited for fast designs typical for the very early stage of the developing process [2, 3]. However, analytical modeling of arbitrary shaped three-dimensional structures is very complicated, if possible at all. Thus, analytical parameter extraction methods have only limited applicability. For final analysis prior to fabrication and for irregular inductor geometries numerical simulation methods normally based on solving the Maxwell equations provide the most accurate characterization. Moreover, the investigated interconnect structure can often be embedded in a small simulation region for which the optimized model of the dominant magnetic field (DMF) can be used even at very high frequencies. The distributed vector and scalar fields must be extracted in structures which may consist of different inhomogeneous complex shaped three-dimensional regions, like splittings, widenings, and vertical connections. As a consequence, the vector and scalar finite element method (FEM) [4] in the frequency domain on unstructured tetrahedral grid $[5,6]$ needs to be addressed.

In this work an optimized model for inductance and resistance analysis of an on-chip inductor at different frequencies is proposed. The model describes the proximity effect and the skin effect typically arising at higher frequencies as well. The three-dimensional FEM simulation software SAP (Smart Analysis Programs) [7] is extended to implement the developed model. Simulation results demonstrate the physical plausibility of the applied model and numerical methods, as well as the necessity of three-dimensional simulations.

\section{THE THEORETICAL BACKGROUND}

If the characteristic lengths of the analyzed structures are much smaller than the considered wave lengths and if for 
conducting areas $\epsilon / \gamma \ll \tau$, then the displacement current can be neglected [8]. $\tau$ is the characteristic period of the time change rate, $\epsilon$ is the characteristic permittivity, and $\gamma$ is the characteristic conductivity. In this case the Maxwell equations can be considerably simplified and for time independent $\mu$ the model DMF is achieved:

$$
\begin{aligned}
& \vec{\nabla} \times \vec{E}=-\mu \partial_{t} \vec{H} \\
& \vec{\nabla} \cdot(\mu \vec{H})=0 \\
& \rho(\vec{\nabla} \times \vec{H})=\vec{E},
\end{aligned}
$$

where $\vec{E}$ is the electric field intensity, $\vec{H}$ is the magnetic field intensity, $\mu$ is the material's permeability, and $\rho$ is the material's electric resistivity. The derivative with respect to time is shortly notated as $\partial_{t}$ instead of $\partial / \partial t$.

\subsection{The Problem Description}

Applying the rotor operator to (3) and substituting the right hand side of (3) by (1) yields the following secondorder differential equation

$$
\vec{\nabla} \times(\rho \vec{\nabla} \times \vec{H})+\mu \partial_{t} \vec{H}=0 .
$$

For the stationary case $\left(\partial_{t} \vec{H}=0\right)$ the finite element analysis of (4) leads to an overdetermined linear equation system [9]. The matrix of this system is positive semi-definite and its zero eigenvalues correspond to the number of tree edges in the graph spanned by the finite element mesh edges [10].

Since $\vec{\nabla} \times \vec{\nabla} \varphi=0$, (4) may be written as

$$
\vec{\nabla} \times[\rho \vec{\nabla} \times(\vec{H}+\vec{\nabla} \varphi)]+\mu \partial_{t} \vec{H}=0,
$$

which leads with the aid of the substitution $\vec{H}_{1}=\vec{H}+\vec{\nabla} \varphi$ to

$$
\vec{\nabla} \times\left(\rho \vec{\nabla} \times \vec{H}_{1}\right)+\mu \partial_{t}\left(\vec{H}_{1}-\vec{\nabla} \varphi\right)=0,
$$

where $\varphi$ is an arbitrary scalar field and $\vec{\nabla} \varphi$ must exist. Equation (6) contains two unknown functions - the vector field $\vec{H}_{1}$ and the scalar field $\varphi$. Thus an additional independent criterion is needed. For numerically stable and unique calculation of $\vec{H}$ it is natural to impose the divergence condition (2), which has not been used till now

$$
\vec{\nabla} \cdot\left[\mu\left(\vec{H}_{1}-\vec{\nabla} \varphi\right)\right]=0 .
$$

Thus the unknown fields $\vec{H}_{1}$ and $\varphi$ are the solution of the partial differential equation system consisting of (6) and (7), which is a boundary value problem numerically calculated by FEM in a simulation domain $\mathcal{V}$ enclosing the investigated structures. The fields are approximated by the following expressions:

$$
\begin{array}{r}
\vec{H}_{1}(\vec{r}, t)=\sum_{j=1}^{n} c_{j}(t) \vec{N}_{j}(\vec{r})+\vec{H}_{K}(\vec{r}, t) \\
\varphi(\vec{r}, t)=\sum_{j=n+1}^{m} c_{j}(t) \lambda_{j}(\vec{r})+\varphi_{K}(\vec{r}, t)
\end{array}
$$

Due to the FEM domain discretization the region of interest $\mathcal{V}$ and its surface $\partial \mathcal{V}$ are subdivided into smaller mesh elements - tetrahedrons and triangles consisting of edge connected nodes. The boundary of the simulation area $\partial \mathcal{V}$ is divided into Dirichlet boundary $\partial \mathcal{V}_{D 1}$ and Neumann boundary $\partial \mathcal{V}_{N 1}$ for $\vec{H}_{1}$ and into Dirichlet boundary $\partial \mathcal{V}_{D 2}$ and Neumann boundary $\partial \mathcal{V}_{N 2}$ for $\varphi$, respectively $\left(\partial \mathcal{V}=\partial \mathcal{V}_{D 1}+\partial \mathcal{V}_{N 1}\right.$ and $\left.\partial \mathcal{V}=\partial \mathcal{V}_{D 2}+\partial \mathcal{V}_{N 2}\right)$. The edges and the nodes in the simulation area are labeled with a set of integers. The nonDirichlet edges are indexed from 1 to $n$ and the non-Dirichlet nodes are indexed from $n+1$ to $m(m>n)$. The non-Dirichlet edges are the edges which do not belong to $\partial \mathcal{V}_{D 1}$, and the non-Dirichlet nodes are the nodes which do not belong to $\partial \mathcal{V}_{D 2}$, respectively. The known function $\vec{H}_{K}$ satisfies the Dirichlet boundary conditions on $\partial \mathcal{V}_{D 1}$ and does not have a tangential component on the non-Dirichlet edges of the simulation area. Analogously the known function $\varphi_{K}$ satisfies the Dirichlet boundary condition on $\partial \mathcal{V}_{D 2}$ and vanishes in the remaining simulation area. $\vec{N}_{j}^{(e)}$ is the Whitney 1-Form vector basis function [11] in a single mesh element and is associated with the $j$-th edge. For example, in a tetrahedral element, if the $j$-th edge belongs to the nodes $p$ and $q$, the corresponding element edge function is:

$$
\vec{N}_{j}^{(e)}=\vec{N}_{p q}^{(e)}=l_{p q}\left(\lambda_{p}^{(e)} \vec{\nabla} \lambda_{q}^{(e)}-\lambda_{q}^{(e)} \vec{\nabla} \lambda_{p}^{(e)}\right),
$$

where $l_{p q}$ is the length of the corresponding edge. The Lagrange interpolation polynomial $\lambda_{j}^{(e)}$ at vertex $j$ in the tetrahedral mesh element is given by:

$$
\lambda_{j}^{(e)}=\frac{1}{4}-\left(\vec{r}-\vec{r}_{B}\right) \cdot \frac{F_{j} \vec{n}_{j}}{3 V},
$$

where $\vec{r}_{B}=\left(\sum_{i=1}^{4} \vec{r}_{i}\right) / 4$ is the barycenter of the tetrahedral mesh element, $\bar{F}_{j}$ is the area of the face opposite to the $j$ th node, $\vec{n}_{j}$ is normal to this face and points outward, and $V$ is the volume of the tetrahedron. The global edge function $\vec{N}_{j}$ consists of all element edge functions belonging to the global edge $j$ and does not have a tangential component on the remaining edges. The global node function $\lambda_{j}$ consists of all element node functions belonging to the global node $j$ and vanishes in the remaining nodes. Consequently the calculated coefficients $c_{j}$ represent the tangential component of the vector field $\vec{H}_{1}$ on the $j$-th edge or the value of the scalar field $\varphi$ on the $j$-th node. The global node or edge functions are "active" only in the neighbor elements of the corresponding node or edge, respectively. Applying the Galerkin method to (6) and (7)

$$
\begin{aligned}
& \int_{\mathcal{V}}\left[\vec{\nabla} \times\left(\rho \vec{\nabla} \times \vec{H}_{1}\right)+\mu \partial_{t}\left(\vec{H}_{1}-\vec{\nabla} \varphi\right)\right] \cdot \vec{N}_{i} d V=0, \\
& i=1 \ldots n \\
& \int_{\mathcal{V}} \vec{\nabla} \cdot\left[\mu\left(\vec{H}_{1}-\vec{\nabla} \varphi\right)\right] \lambda_{i} d V=0, i=n+1 \ldots m
\end{aligned}
$$

and using the first vector Green's theorem and the first scalar Green's theorem the following system is obtained:

$$
\begin{aligned}
& \int_{\mathcal{V}}\left(\vec{\nabla} \times \vec{N}_{i}\right)\left(\rho \vec{\nabla} \times \vec{H}_{1}\right) d V+\int_{\mathcal{V}} \mu \partial_{t} \vec{H}_{1} \cdot \vec{N}_{i} d V- \\
& -\int_{\partial \mathcal{V}} \vec{n} \cdot\left[\vec{N}_{i} \times\left(\rho \vec{\nabla} \times \vec{H}_{1}\right)\right] d A-\int_{\mathcal{V}} \mu \partial_{t} \vec{\nabla} \varphi \cdot \vec{N}_{i} d V=0, \\
& i=1 \ldots n \\
& \quad \int_{\mathcal{V}} \vec{\nabla} \lambda_{i} \cdot\left(\mu \vec{H}_{1}\right) d V-\int_{\mathcal{V}} \vec{\nabla} \lambda_{i} \cdot(\mu \vec{\nabla} \varphi) d V- \\
& -\int_{\partial \mathcal{V}} \lambda_{i} \vec{n} \cdot\left[\mu\left(\vec{H}_{1}-\vec{\nabla} \varphi\right)\right] d A=0, \quad i=n+1 \ldots m
\end{aligned}
$$

The correct gradient-field-free magnetic field intensity $\vec{H}$ and the current density distribution $\vec{J}$ are calculated from:

$$
\vec{J}=\vec{\nabla} \times \vec{H}=\vec{\nabla} \times \vec{H}_{1}, \vec{H}=\vec{H}_{1}-\vec{\nabla} \varphi
$$




\subsection{Boundary Conditions}

The boundary term in (12) can be expressed as follows:

$$
\begin{aligned}
& \int_{\partial \mathcal{V}} \vec{n} \cdot\left[\vec{N}_{i} \times\left(\rho \vec{\nabla} \times \vec{H}_{1}\right)\right] d A=\int_{\partial \mathcal{V}} \vec{n} \cdot\left(\vec{N}_{i} \times \vec{E}\right) d A= \\
& \int_{\partial \mathcal{V}}\left(\vec{n} \times \vec{N}_{i}\right) \cdot \vec{E} d A=\int_{\partial \mathcal{V}}(\vec{E} \times \vec{n}) \cdot \vec{N}_{i} d A
\end{aligned}
$$

with $\vec{E}=\rho \vec{J}=\rho \vec{\nabla} \times \vec{H}_{1} . \vec{N}_{i}$ has a tangential component only on the $i$-th edge. For $i=1$. .n the $i$-th edge is a non-Dirichlet edge. Thus $\vec{N}_{i}$ has no tangential component on the edges of the Dirichlet boundary $\partial \mathcal{V}_{D 1}$ and must be perpendicular to $\partial \mathcal{V}_{D 1}$ (or parallel to $\vec{n}$ ). As clearly shown by the third member of (15) the boundary integral in (12) has a contribution only for the Neumann boundary $\partial \mathcal{V}_{N 1}: \int_{\partial \mathcal{V}_{N 1}}(\vec{E} \times \vec{n}) \cdot \vec{N}_{i} d A$. Only the supply parts of the wire, which are used to force the electric current, lie directly on the boundary faces of the simulation area. The remaining parts of the wire are surrounded by dielectric material. In this work the dielectric environment enclosing the wire is assumed to be sufficiently thick so that $\vec{E}$ could be neglected on the dielectric outer bounds of the simulation domain. On the other hand the electric current density is forced in a direction perpendicular to the conductor boundary faces. Thus $\vec{E}$ will be also perpendicular to these faces and the boundary term in (12) will be zero.

The supplied total current in the inductor wire is considered by the Dirichlet condition for $\vec{H}_{1}$ :

$$
I=\oint_{\partial \mathcal{A}} \vec{H}_{1} \cdot d \vec{r}=\oint_{\partial \mathcal{A}} \vec{H} \cdot d \vec{r} \simeq H_{t} \sum_{i=0}^{p} l_{i},
$$

where $\partial \mathcal{A}$ is an arbitrary closed loop around the conducting wire, $p$ is the number of Dirichlet edges, which build this loop, and $l_{i}$ is the length of the $i$-th loop edge. If the Dirichlet edges are labeled with integers from $m+1$ to $k$ $(k>m)$, the Dirichlet boundary function $\vec{H}_{K}$ from (8) is expressed as $\vec{H}_{K}=\sum_{j=m+1}^{k} c_{j} \vec{N}_{j}$ with $c_{j}=H_{t}$ for $j=m+1 \ldots k$ and $p=k-m$.

Analogously the boundary term in (13) can be expressed as

$$
\int_{\partial \mathcal{V}} \lambda_{i} \vec{n} \cdot\left[\mu\left(\vec{H}_{1}-\vec{\nabla} \varphi\right)\right] d A=\int_{\partial \mathcal{V}} \lambda_{i} \vec{n} \cdot \vec{B} d A=\int_{\partial \mathcal{V}_{N 2}} \lambda_{i} \vec{n} \cdot \vec{B} d A
$$

with $\vec{B}=\mu \vec{H}=\mu\left(\vec{H}_{1}-\vec{\nabla} \varphi\right) . \quad \vec{B}$ is assumed to be perpendicular to $\vec{n}$ on the Neumann boundary $\partial \mathcal{V}_{N 2}$. Thus the boundary integral in (13) vanishes.

Dirichlet boundary conditions must be given also for $\varphi$. The Dirichlet nodes are labeled with numbers from $k+1$ to $l$ $(l>k)$. The function $\varphi_{K}$ from (9) can analogously be defined as $\varphi_{K}=\sum_{j=k+1}^{l} c_{j} \lambda_{j}$. For the calculations it is sufficient that one node of the simulation domain is forced as Dirichlet node. Thus, in this specific case $\varphi_{K}=c_{k+1} \lambda_{k+1}$. As far as only $\vec{\nabla} \varphi$ is required and not $\varphi$, the coefficient $c_{k+1}$ of this Dirichlet node can be set to an arbitrary value.

\subsection{Assembling the Matrix and the Right Hand Side Vector}

Under consideration of (8), (9) and the above specified boundary conditions the weighted equation system (12) and (13) corresponds in the frequency domain with

$$
\left[\begin{array}{ll}
{[A]} & {[B]} \\
{[B]^{T}} & {[C]}
\end{array}\right] \quad\{c\}=\{b\},
$$

$$
\begin{aligned}
& A_{i j}=\int_{\mathcal{V}}\left(\vec{\nabla} \times \vec{N}_{i}\right) \cdot\left(\rho \vec{\nabla} \times \vec{N}_{j}\right) d V+\jmath \omega \int_{\mathcal{V}} \vec{N}_{i} \cdot\left(\mu \vec{N}_{j}\right) d V \\
& i=1 \ldots n, j=1 \ldots n \\
& B_{i j}=-\jmath \omega \int_{\mathcal{V}} \vec{N}_{i} \cdot\left(\mu \vec{\nabla} \lambda_{j}\right) d V, \quad i=1 \ldots n, j=n+1 \ldots m \\
& C_{i j}=\jmath \omega \int_{\mathcal{V}} \vec{\nabla} \lambda_{i} \cdot\left(\mu \vec{\nabla} \lambda_{j}\right) d V, i=n+1 \ldots m, j=n+1 \ldots m \\
& b_{i}=\jmath \omega \sum_{j=k+1}^{l} c_{j} \int_{\mathcal{V}} \vec{N}_{i} \cdot\left(\mu \vec{\nabla} \lambda_{j}\right) d V-\jmath \omega \sum_{j=m+1}^{k} c_{i} \int_{\mathcal{V}} \vec{N}_{i} \cdot\left(\mu \vec{N}_{j}\right) d V- \\
& -\sum_{j=m+1}^{k} c_{j} \int_{\mathcal{V}}\left(\vec{\nabla} \times \vec{N}_{i}\right) \cdot\left(\rho \vec{\nabla} \times \vec{N}_{j}\right) d V, i=1 \ldots n \\
& b_{i}=\jmath \omega \sum_{j=m+1}^{k} c_{j} \int_{\mathcal{V}} \vec{\nabla} \lambda_{i} \cdot\left(\mu \vec{N}_{j}\right) d V-\jmath \omega \sum_{j=k+1}^{l} c_{j} \int_{\mathcal{V}} \vec{\nabla} \lambda_{i} \cdot\left(\mu \vec{\nabla} \lambda_{j}\right) d V, \\
& i=n+1 \ldots m \text {, }
\end{aligned}
$$

where the time convention $e^{\jmath \omega t}$ is used and suppressed. To obtain a symmetric equation system (13) has been multiplied by $-\jmath \omega$, where $\omega$ is the angular frequency.

\subsection{Inductance and Resistance Extraction}

The inductance and the resistance are calculated by the magnetic energy and by the electric power, respectively.

$$
L=\frac{1}{I^{2}} \int_{\mathcal{V}} \mu H^{2} d V, R=\frac{1}{I^{2}} \int_{\mathcal{V}}\left(\vec{\nabla} \times \vec{H}_{1}\right) \cdot\left(\rho \vec{\nabla} \times \vec{H}_{1}\right) d V .
$$

$I$ is the total current in the inductor, which defines the Dirichlet boundary (16) for $\vec{H}_{1}$ and $\vec{H}$ is given by (14).

\section{DOMAIN DISCRETIZATION}

The domain discretization is the first step of the finite element analysis. For this purpose the entire domain is divided into smaller sub-domains, called elements. For threedimensional problems the volume elements can be rectangular bricks or tetrahedrons, for instance. Then the boundary surface is broken up into rectangles or triangles, respectively. The rectangular elements are perfectly suited for discretizing rectangular domains with an uniform density. However, the physical models can not always be limited to specific regular geometries. The mesh density must be high enough to achieve sufficiently accurate solutions. Unfortunately a high number of mesh elements leads to many unknowns, causing high memory demands and long simulation times. For this purpose it is required to keep the element size as large as possible for a desired accuracy. It is desirable to use a finer mesh (or smaller elements) only in the regions where high field dynamic is anticipated. In this work the regions are described by tetrahedral or triangular elements, depending on the discretized object - volume or boundary surface. In addition, not only the density but also the quality of the tetrahedral elements affects directly the FEM accuracy and efficiency [12].

The example inductor geometry presented in the next section is described by the constructive solid geometry (CSG) format. It is discretized with the three-dimensional tetrahedron mesh generation software Netgen [13]. The CSG format is very convenient for the description of small or medium size 
structures like the spiral inductor presented in the example section. The geometry is defined by Eulerian operations (union, intersection, and complement) from primitives. The primitives are generic volume elements like cubes, cylinders, spheres, or even half-spaces defined by an arbitrary point in the boundary plane and an outward normal vector. If CSG input is used, Netgen starts with the computation of the corner points. Then the edges are defined and meshed into segments. Next, the faces are generated by an advancing front algorithm [14]. After optimization of the surface mesh the volume inside is filled with tetrahedrons by a fast Delaunay algorithm [15]. Finally the volume mesh is optimized.

\section{EXAMPLES AND RESULTS}

As example a typical on-chip spiral inductor structure as discussed in [16] is investigated. The simulation domain consists of a transparent insulating rectangular brick over an opaque substrate brick as shown in Fig. 1. The aluminum inductor is placed in the insulating environment about $5 \mu \mathrm{m}$ above the substrate area. The substrate is assumed to have a constant resistivity of $10 \Omega \mathrm{cm}$. The cross-section of the conductor is $20 \mu \mathrm{m} \times 1.2 \mu \mathrm{m}$. The horizontal distance between the winding wires is $10 \mu \mathrm{m}$. The outer dimensions of the inductor are $300 \mu \mathrm{m} \times 300 \mu \mathrm{m}$. The inductor is completely surrounded by the dielectric environment, except of the two small delimiting faces which lie directly in the boundary planes of the simulation domain. The conductor area, the dielectric, and the substrate area close to the conductor are discretized much finer then the remaining simulation domain. This is shown in Fig. 2 where a part of the dielectric environment is removed to visualize in detail the generated mesh inside the simulation domain. The variation of the fields in the finer discretized areas is expected to be much higher than in the coarser discretized domain. This special discretization reduces the number of generated nodes and edges, and the number of the linear equations respectively, even for big simulation environments which have to be used to satisfy the assumption of the zero Neumann boundary condition. Of coarse such a discretization is only possible, if an unstructured mesh is used.

The current density distribution depends heavily on the operating frequency in the analyzed frequency domain. It is unknown and arises from the simulation. At the beginning of the simulation only the total current in the inductor is known. As mentioned above it is set by the Dirichlet boundary condition for $\vec{H}_{1}$ which is given by the tangential component of the magnetic field intensity $H_{t}$ on the element edges, surrounding one of the conductor faces lying on the outer bound of the simulation domain.

The resistance and inductance values of the structure of interest are calculated numerically at different frequencies with and without the substrate influence. The corresponding results are presented in Table 1 . While the inductance decreases slowly with increasing operating frequency, the resistance rises dramatically, which matches well the observed current density distribution and the skin effect, respectively. A surface view of the current density distribution in the conductor is shown in Fig. 3 and Fig. 4 for $100 \mathrm{MHz}$ and $10 \mathrm{GHz}$, respectively. At $100 \mathrm{MHz}$ the skin depth is about $6 \mu \mathrm{m}$ and nearly the whole conductor cross-section is filled up by the current. At $10 \mathrm{GHz}$ the skin depth is about $0.6 \mu \mathrm{m}$ and the current is concentrated at the vertical side walls of the conductor. Fig. 5 shows the spatial current density distribution
Table 1: Calculated inductance and resistance.

\begin{tabular}{c||c||c||c}
\hline $\mathbf{f}[G H z]$ & $\begin{array}{c}\mathbf{L}[n H] \text { without } \\
\text { substrate }\end{array}$ & $\begin{array}{c}\mathbf{L}[n H] \text { with } \\
\text { substrate }\end{array}$ & $\mathbf{R}[\Omega]$ \\
\hline \hline 0.001 & 2.6887 & 2.6881 & 3.127 \\
\hline \hline 0.01 & 2.6887 & 2.6877 & 3.127 \\
\hline \hline 0.1 & 2.688 & 2.688 & 3.132 \\
\hline \hline 1 & 2.6516 & 2.6514 & 3.463 \\
\hline \hline 10 & 2.5501 & 2.5493 & 5.396 \\
\hline \hline 100 & 2.5458 & 2.5457 & 13.156 \\
\hline \hline
\end{tabular}

at $1 \mathrm{GHz}$ as directed cones placed in the discretization nodes inside of the conductor area. The cone's size and darkness are proportional to the field strength. Fig. 6 depicts the corresponding spatial distribution of the magnetic field inside the dielectric environment around the inductor. Fig. 7 and Fig. 8 show the very different current density distribution close to the small via for $100 \mathrm{MHz}$ and $10 \mathrm{GHz}$, respectively. Fig. 9 and Fig. 10 show the current density distribution and the corresponding magnetic field intensity in the substrate at $1 \mathrm{GHz}$. The underlying substrate does not influence the inductance and the resistance of the inductor, because of the relative high substrate resistivity. The values of the current density distribution in the substrate (Fig. 9) differ vastly to those in the inductor (Fig. 3 and Fig. 4). As shown in Table 1 the calculated inductance taking into account the influence of the substrate does not differ from the inductance without substrate influence.

As the Q-factor of an inductor is inversely proportional to its resistance, making the inductor wire thicker might decrease the resistance and increase the Q-factor. However, as the examples show this is not the case for high frequencies at which the skin effect is noticeable. In these cases the current flows only in the area very close to the vertical surface and a wider transversal conductor cross section would not change the situation. For the visualization VTK [17] is used.

\section{CONCLUSION}

In this work a new method for inductance and resistance parameter extraction is proposed. It is assumed that the operating frequencies and the characteristic lengths of the investigated structures are suitable to use the DMF model. This is typically the case for on-chip inductors and interconnect loops. The rotor operator which appears in the second order partial differential equation for the magnetic field $\vec{H}$ allows arbitrary unknown gradient fields $\vec{\nabla} \varphi$. The obtained partial differential equation system is solved numerically by FEM. Instead of solving the more complicated wave equation, considering DMF, it is sufficient to solve the diffusion equation to investigate the parameters and field distributions of interest, which is of course the more efficient way.

\section{REFERENCES}

[1] Y. Cao, X. Huang, D. Sylvester, T.-J. King, and C. Hu. Impact of on-chip interconnect frequency-dependent $\mathrm{R}(\mathrm{f}) \mathrm{L}(\mathrm{f})$ on digital and $\mathrm{RF}$ design. In Proc. IEEE International ASIC-SoC Conference, September 2002. 


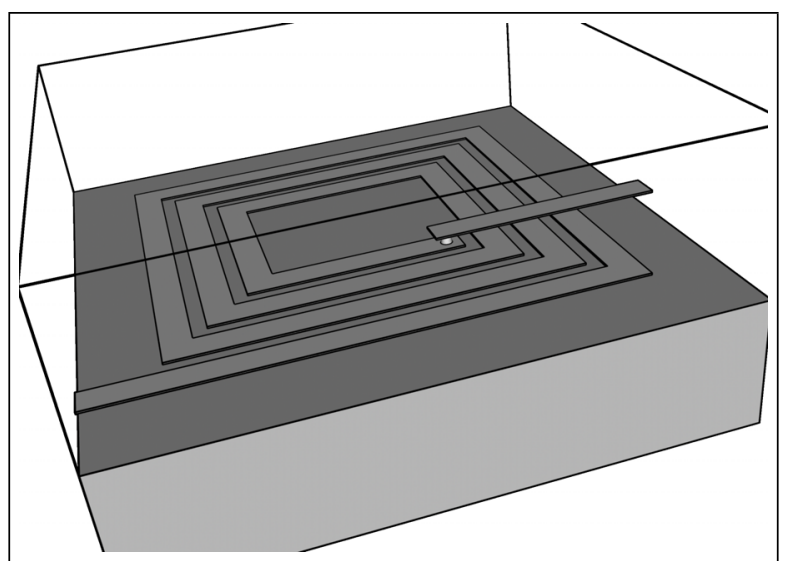

Figure 1: The simulation domain.

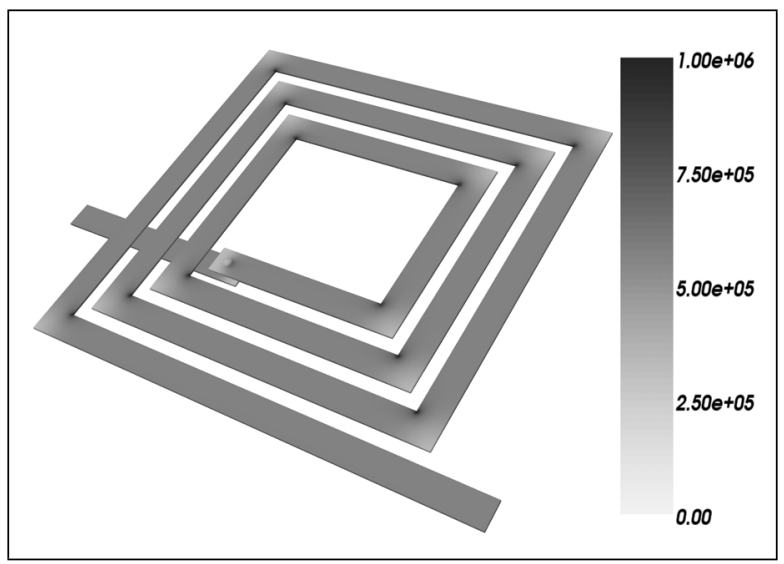

Figure 3: Surface view of the current density $\left[\mathrm{A} / \mathrm{m}^{2}\right]$ distribution at $100 \mathrm{MHz}$.

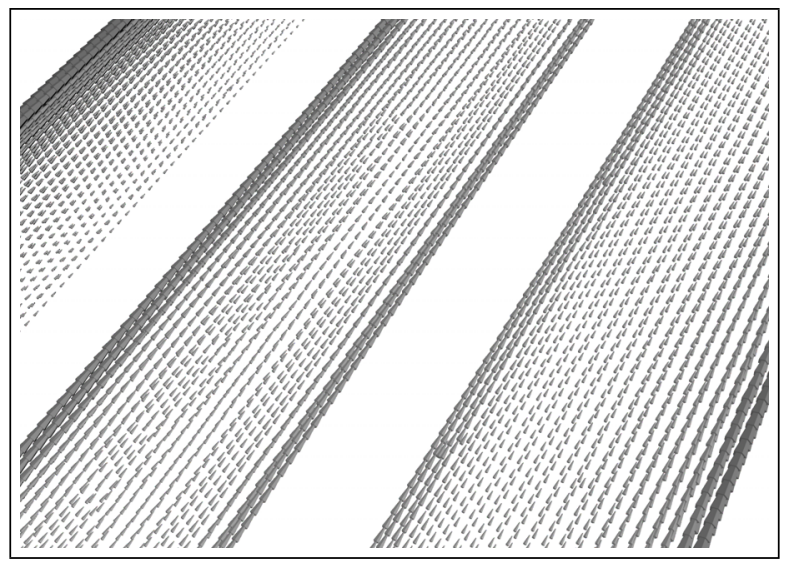

Figure 5: Current density distribution at $1 \mathrm{GHz}$.

[2] A. Nieuwoudt and Y. Massoud. Variability-aware multilevel integrated spiral inductor synthesis. IEEE Trans. Comput.-Aided Design Integr. Circuits Syst., 25(12):2613-2625, December 2006.

[3] F. Y. Huang, J. X. Lu, D. M. Jiang, X. C. Wang, and N. Jiang. A novel analytical approach to parameter

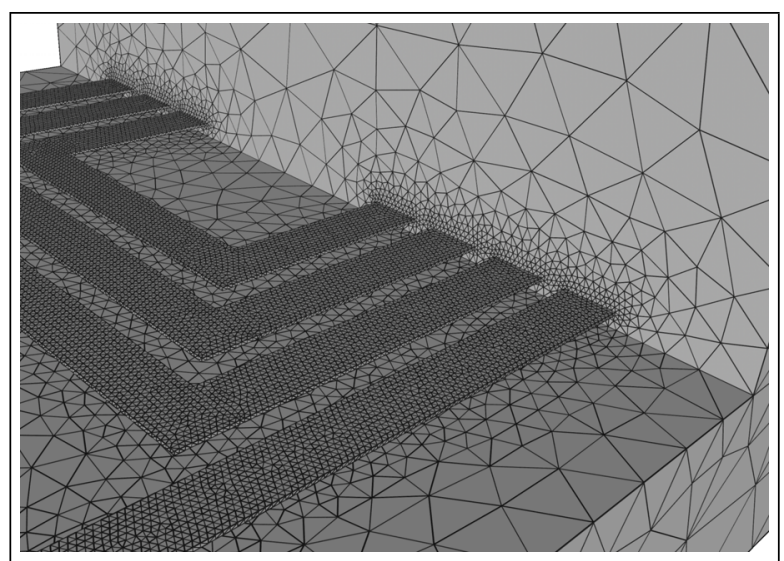

Figure 2: The generated grid.

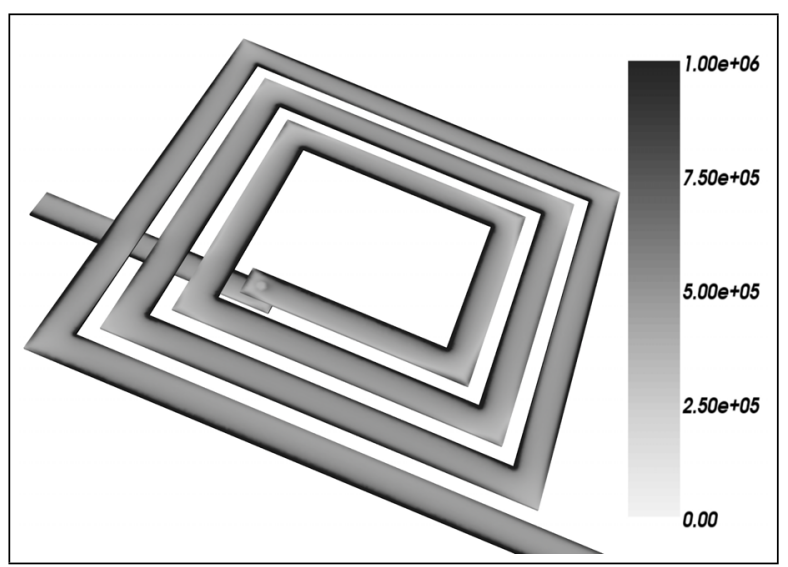

Figure 4: Surface view of the current density $\left[A / m^{2}\right]$ distribution at $10 \mathbf{G H z}$.

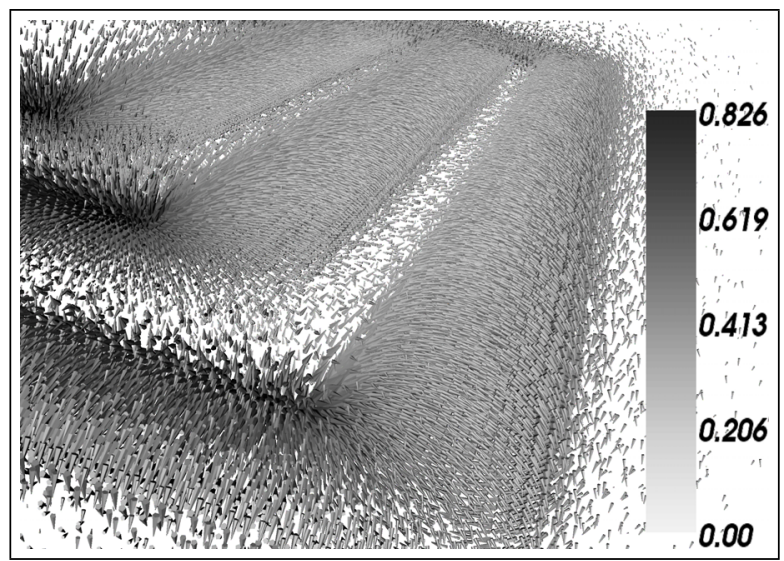

Figure 6: Magnetic field intensity $[A / m]$ at $1 \mathrm{GHz}$.

extraction for on-chip spiral inductors taking into account high-order parasitic effect. Solid-State Electronics, 50(9-10):1557-1562, September-October 2006.

[4] J. Jin. The Finite Element Method in Electromagnetics. John Wiley and Sons, New York, 2002. 


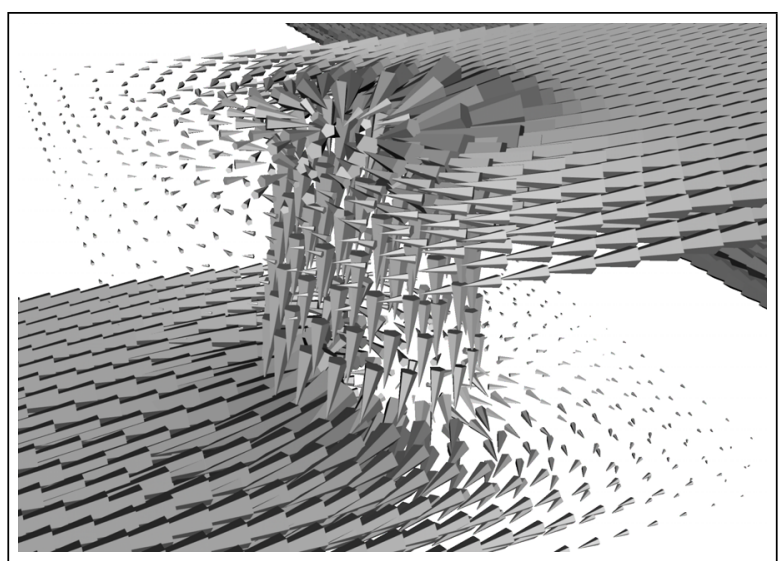

Figure 7: Current density distribution in the via at $100 \mathrm{MHz}$.

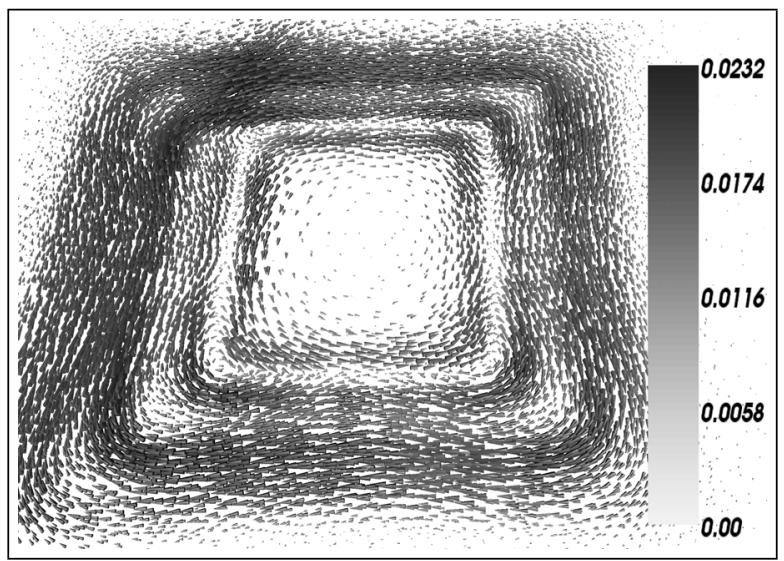

Figure 9: Current density $\left[A / \mathrm{m}^{2}\right]$ distribution in the substrate at $1 \mathrm{GHz}$.

[5] P. Fleischmann and S. Selberherr. A new approach to fully unstructured three-dimensional Delaunay mesh generation with improved element quality. In Proc. Simulation of Semiconductor Processes and Devices, pages 129-130, 1996.

[6] P. Fleischmann, R. Sabelka, A. Stach, R. Strasser, and S. Selberherr. Grid generation for three-dimensional process and device simulation. In Proc. Simulation of Semiconductor Processes and Devices, pages 161-166, 1996.

[7] R. Sabelka and S. Selberherr. SAP - A program package for three-dimensional interconnect simulation. In Proc. Intl. Interconnect Technology Conference, pages 250-252, Burlingame, California, 1998.

[8] A. Prechtl. Vorlesungen über Theoretische Elektrotechnik. Institut für Grundlagen umd Theorie der Elektrotechnik, TU Wien, 1996. Zweiter Teil: Elektrodynamik.

[9] P. Meuris, W. Schoenmaker, and W. Magnus. Strategy for electromagnetic interconnect modeling. IEEE Trans. Comput.-Aided Design Integr. Circuits Syst., 20(6):753-762, June 2001.

[10] O. Biró, K. Preis, and K. R. Richter. On the use of the magnetic vector potential in the nodal and edge

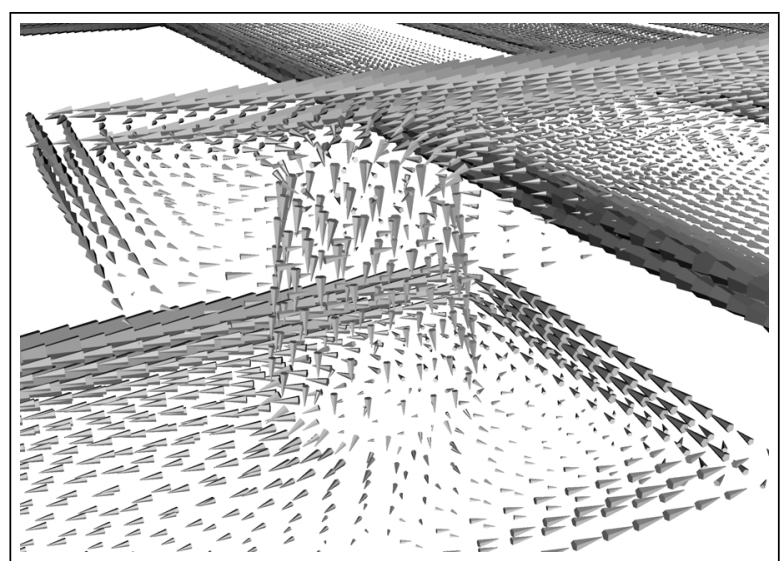

Figure 8: Current density distribution in the via at $10 \mathrm{GHz}$.



Figure 10: Magnetic field intensity $[A / m]$ in the substrate at $1 \mathrm{GHz}$.

finite element analysis of 3D magnetostatic problems. IEEE Trans. Magn., 32(3):651-654, May 1996.

[11] H. Whitney. Geometric Integration Theory. Princeton, NJ:Princeton University Press, 1957.

[12] M. Dorica and D. Giannacopoulos. Impact of mesh quality improvement systems on the accuracy of adaptive finite-element electromagnetics with tetrahedra. IEEE Trans. Magn., 41(5):1692-1695, May 2005.

[13] J. Schöberl. Netgen - Automatic mesh generator. http://www.hpfem.jku.at/netgen.

[14] J. Schöberl. Netgen - An advancing front 2d/3d-mesh generator based on abstract rules. Computing and Visualization in Science, 1(1):41-52, July 1997.

[15] H. Borouchaki and S. H. Lo. Fast Delaunay triangulation in three dimensions. Computer methods in applied mechanics and engineering, 128(1):153-167, December 1995.

[16] Y. Zhan, R. Harjani, and S. Sapatnekar. On the selection of on-chip inductors for the optimal VCO design. In Custom Integrated Circuits Conference, pages 277-280, Oct. 2004.

[17] W. Schroeder, K. Martin, and B. Lorensen. The Visualization Toolkit. Kitware, Inc., USA, 2004. 